gastroscopy using gaze control and targeting ten points scattered through the stomach (Figure 1b).

Results Four expert endoscopists and one novice used gaze control to successfully navigate a gastroscope through the simulated UGIT. All were able to independently intubate the oesophagus and accurately locate ten targets placed in the fundus, body, antrum and pylorus of the stomach without touching the endoscope.

Conclusions Gaze control endoscopy is a feasible concept. It allows ergonomic, user-friendly and intuitive control whilst maintaining the benefits of a flexible endoscope.

\section{PTH-052 DOES THROAT SPRAY IN COMBINATION WITH INTRAVENOUS SEDATION/ANALGESIA FOR ELECTIVE GASTROSCOPY INCREASE RESPIRATORY COMPLICATIONS?}

${ }^{1}$ Mehul Patel*, ${ }^{1}$ Abisoye Akintimehin, ${ }^{1}$ Nishmi Gunasingam, ${ }^{2}$ Mayur Kumar, ${ }^{1}$ Amyn Haji, ${ }^{1}$ Bu'Hussain Hayee. 'King's College Hospital NHS Foundation Trust, London, UK; ${ }^{2}$ Princess Royal University Hospital, Kent, UK

\subsection{6/gutjnl-2019-BSGAbstracts.77}

Introduction Safe sedation practice is a recognised cornerstone of high-quality endoscopy. There are concerns that local anaesthetic throat spray (TS) in combination with intravenous sedative and analgesic agents (ISAAs) can precipitate respiratory complications, specifically, aspiration pneumonia. Current BSG standards for upper gastrointestinal endoscopy recommend 'caution should be exercised' in using agents combined with TS but acknowledges the paucity of evidence for this, with the few relevant studies being performed several decades ago.

Methods A retrospective, two-centre cohort study was performed. Only diagnostic, outpatient gastroscopies (OGDs) performed 2013-2018 were reviewed. Patients residing in a postcode region (definite or possible) outside of the catchment of the two centres were excluded from final analysis. Endoscopy reports and electronic patient records were reviewed to identify all patients who presented to hospital within 30 days of their procedure to determine the underlying reason for this.

Results 5,803 OGDs met the inclusion criteria. 148 (2.6\%) patients presented to hospital within 30 days of procedure, 17 $(0.3 \%)$ were due to potential respiratory complications (pneumonia, respiratory tract infection and infective exacerbation of COPD). Choice of drug included: TS 3163 (54.5\%); TS + middazolam 1508 (26\%); TS +fentanyl 28 (0.5\%); TS+dual agent $382(6.6 \%)$ and non-TS $722(12.4 \%)$. Only TS and TS+anallgesic/sedative groups were compared. There was no significant difference in the rate of respiratory complications between the two groups $(p=1.0$, two-sided Fisher's Exact Test). 12/17 respiratory complications occurred in the TS group. Procedure discomfort scores were similar in this group when compared to the TS and additional agent group.

Conclusions While safe sedation practice should remain the cornerstone of management and clinicians should continue to exercise caution in patients with respiratory comorbidities, inpatients and therapeutic procedures, the combination of TS
+ISAAs does not increase the risk of respiratory complications following elective diagnostic OGD.

\section{PTH-053 VENTING PERCUTANEOUS ENDOSCOPIC GASTROSTOMY (VPEG): SIX YEAR EXPERIENCE FROM A TERTIARY REFERRAL CENTRE}

${ }^{1}$ Ross J Porter*, ${ }^{2}$ Gillian Leggett, ${ }^{2}$ Alistair McKinlay, ${ }^{2}$ Emma L Metcalfe. ${ }^{1}$ School of Medicine Medical Sciences and Nutrition, University of Aberdeen; ${ }^{2}$ Department of Digestive Disorders, Aberdeen Royal Infirmary

\subsection{6/gutjnl-2019-BSGAbstracts.78}

Introduction Chronic nausea, vomiting and pain are all well recognised to negatively impact on health-related quality of life. In patients with gastrointestinal obstruction, gastric decompression via venting gastrostomy can offer symptomatic relief. However, the benefits must be considered against the risks associated with the invasive procedure, especially in malignant disease with a limited prognosis. Data on this important and largely uncharacterised cohort are sparse. We describe this population and outcome data from our specialist centre over a six-year period.

Methods Patients undergoing vPEG insertion between 20122018 were prospectively identified from a designated PEG database. Data were extracted from medical records with last follow-up November 2018. Analysis was descriptive. Caldicott Guardian approval was granted.

Results Nineteen patients (male $n=11$, female $n=8$ ) were identified; mean age 59.9 years (range 18-90). Fourteen had malignant obstruction secondary to locally advanced or metastatic cancer from upper gastrointestinal $(n=8,57 \%)$, colorectal $(n=5,36 \%)$, or gynaecological $(n=1,7 \%)$ sites. Five had benign obstruction from post-operative complications $(n=3$, $60 \%)$, gastric outlet obstruction $(n=1,20 \%)$, or connective tissue sequelae $(n=1,20 \%)$. Tube insertion was successful in eighteen (95\%) patients, discussed hereafter. There were no procedure related complications of bleeding, perforation or peritonitis (as defined on CT or requiring surgery). No patients had post-procedure pain significant enough to require CT imaging within the first seven days. However, one patient $(5.6 \%)$ with malignancy sustained a confirmed perforation during secondary placement of a jejunal extension tube for feeding. In three benign cases (60\%) versus zero malignant cases, the gastrostomy had been removed during the follow-up period. Benign indications were associated with better survival outcomes compared to malignant disease (mean 795 days/ range $107-1917+$ and 60.4 days/ range 2-258, respectively). From data available $(n=10)$, all patients reported resolution or improvement of nausea, vomiting or pain.

Conclusions Our data supports the use of vPEG as a safe and effective palliative intervention in both benign and malignant gastrointestinal obstruction. A defined strategy for the assessment of such patients is lacking and development of an agreed clinical pathway may streamline and improve patient care. Important such considerations would include up-to-date imaging to delineate anatomy, and prior gastric decompression with a wide bore nasogastric tube to reduce the risks of aspiration and peritonitis. 